\title{
Morphologie dentaire comparée de Pitymys lusitanicus et Pitymys duodecimcostatus (Arvicolidae, Rodentia) dans le nord-ouest de l'Espagne
}

\author{
par P. BRUNET-LECOMTE ', G. BROCHET ',
} J. CHALINE ${ }^{1,2}$ et M. DELIBES ${ }^{3}$

'EPHE, Laboratoire de Préhistoire et Paléoécologie du Quaternaire, 6, bd. Gabriel, 21100 Dijon, France.

${ }^{2}$ U.A. CNRS 157, Centre de Géodynamique sédimentaire et Evolution géobiologique, 6, bd. Gabriel, 21100 Dijon, France.

${ }^{3}$ Estacion Biologica de Doñana, Apdo 1056, 41080 Sevilla, Espagne

Summary. - Lower $\mathbf{M}_{1}$ morphological comparisons between Pitymys lusitanicus and Pitymys duodecimcostatus from North-Western Spain by the mean of multivariate analysis (13 variables deduced from 23 teeth measures) show that intraspecific variability is greater than interspecific one. When $P$. lusitanicus is allopatric with $P$. duodecimcostatus, their $\mathbf{M}_{1}$ are more variable, some of them looking as $P$. duodecimcostatus. This morphological displacement may be related either to a larger ecological niche or to interspecific competition with Microtus species. Pitymys lusitanicus appears to be derived from P. duodecimcostatus.

Résumé. - Cette étude a pour objet de comparer la morphologie dentaire des $\mathbf{M}_{1}$ inférieures de Pitymys lusitanicus lorsqu'il est sympatrique ou allopatrique avec Pitymys duodecimcostatus dans le nord-ouest de l'Espagne. Cette comparaison faite sur du matériel actuel, bien identifié spécifiquement, a été réalisée à l'aide des analyses statistiques multivariées portant sur treize variables calculées à partir de vingt-trois mesures prises sur chaque dent. Lorsque $P$. lusitanicus est allopatrique avec $\boldsymbol{P}$. duodecimcostatus, il présente une variabilité morphologique accrue de la $\mathbf{M}_{1}$, certaines dents pouvant présenter une morphologie semblable à celle de $P$. duodecimcostatus. Lorsque $P$. lusitanicus est sympatrique avec $P$. duodecimcostatus, sa variabilité morphologique est beaucoup plus restreinte et ne mime plus celle de $P$. duodecimcostatus. Ce déplacement de morphologie pourrait résulter du fait que $P$. lusitanicus occupe une plus large niche écologique en zone allopatrique. Cette étude a des applications paléontologiques et évolutives. Elle montre notamment la difficulté d'utiliser les $\mathrm{M}_{1}$ pour distinguer les Pitymys fossiles sans l'utilisation des analyses multivariées. Elle suggère que Pitymys lusitanicus est une forme dérivée de $P$. duodecimcostatus au cours du Quaternaire.

\section{I - INTRODUCTION}

Ellerman et Morrison-Scott (1951) reconnaissaient l'existence de deux espèces de Pitymys dans le nord-ouest de la péninsule ibérique, Pitymys savii (de SélysLongchamps, 1838) et Pitymys duodecimcostatus (de Sélys-Longchamps, 1839).

Mammalia, t. $51, n^{\circ} 1,1987$. 
P. savii comprenait les sous-espèces pyrenaicus de Sélys-Longchamps, 1847 (Pyrénées), lusitanicus Gerbe, 1879 (Portugal), mariae F. Major, 1905 (Galice), pelandonius Miller, 1908 (province de Burgos). $P$. duodecimcostatus était représenté par une seule sous-espèce $P$. $d$. centralis Miller, 1908 dans la province de Burgos. Heim de Balsac et de Beaufort (1967) mettent en évidence la sympatrie des formes pyrenaicus et mariae le long d'une zone allant des Asturies au Béarn, et démontrent ainsi qu'elles ne peuvent appartenir à la même espèce! Almaça (1967) montre à partir de l'étude de la denture que $P$. lusitanicus, tout comme $P$. mariae, se range dans le groupe "duodecimcostatus" et non dans le groupe "saviipyrenaicus ». Les études caryologiques de Meylan (1970), Winking et Niethammer (1970) confirment ces hypothèses en montrant que $P$. lusitanicus, incluant $P$. mariae et $P$. duodecimcostatus, ont le même nombre diploïde de chromosomes $(2 \mathrm{~N}=62)$, tandis que $P$. pyrenaicus a le même nombre que $P$. savii d'Italie $(2 \mathrm{~N}=54)$.

Si les cartes de répartition de $P$. duodecimcostatus et $P$. lusitanicus (Almaça 1973 ; Niethammer et Krapp 1982) montrent une distribution largement allopatrique des deux espèces, elles présentent cependant une zone de sympatrie en Vieille Castille et León.

Le but de ce travail est de comparer la morphologie dentaire des $M_{1}$ de $P$. lusitanicus et de $P$. duodecimcostatus recueillis dans le nord-ouest de l'Espagne en zone d'allopatrie et de sympatrie. Nous nous intéressons essentiellement d'une part à la comparaison intraspécifique chez $P$. lusitanicus et d'autre part à la comparaison interspécifique des deux espèces.

\section{II - MATÉRIEL ET LOCALiSATION}

Le matériel provient de six stations (fig. 1), quatre de Vieille Castille : Quintana del Puente, Santa Maria del Campo, Urbel del Castillo, Lomilla de Pisuerga, et deux de Galice : Verin et Sarreaus. Pour chacune de ces stations on a représenté (fig. 1) les pourcentages des principaux groupes de micromammifères (Soricidae, Muridae, Arvicolidae, Pitymys) trouvés dans les lots de pelotes d'où a été extrait le matériel. Le type de climat, l'altitude, la température moyenne de janvier, la température moyenne de juillet et la précipitation totale annuelle sont donnés pour chaque station dans le tableau 1. Pour les variables climatiques on a associé la station météorologique la plus proche de la localité considérée, soit : Palacios de Alcor pour Quintana del Puente, Lerma pour Santa Maria del Campo, Aguilar de Campo pour Urbel et Lomilla, Ginzo de Limia pour Verin et Sarreaus.

Le matériel est constitué de $338 \mathrm{M}_{1}$ de Pitymys trouvées dans les pelotes de réjection de chouette effraie, Tyto alba, provenant des stations décrites précédemment. L'identification des deux espèces $P$. lusitanicus et $P$. duodecimcostatus a été faite à partir de l'examen du crâne (proodonte chez $P$. duodecimcostatus, orthodonte chez $P$. lusitanicus) et de quelques mesures crâniennes (longueur du diastème supérieur, de la rangée dentaire, etc.) proposées par Spitz (1978). Seul $P$. lusitanicus a été reconnu à Verin, Sarreaus, Urbel et Lomilla, tandis qu'à Quintana et Santa Maria del Campo $P$. lusitanicus et $P$. duodecimcostatus sont sympatriques. 


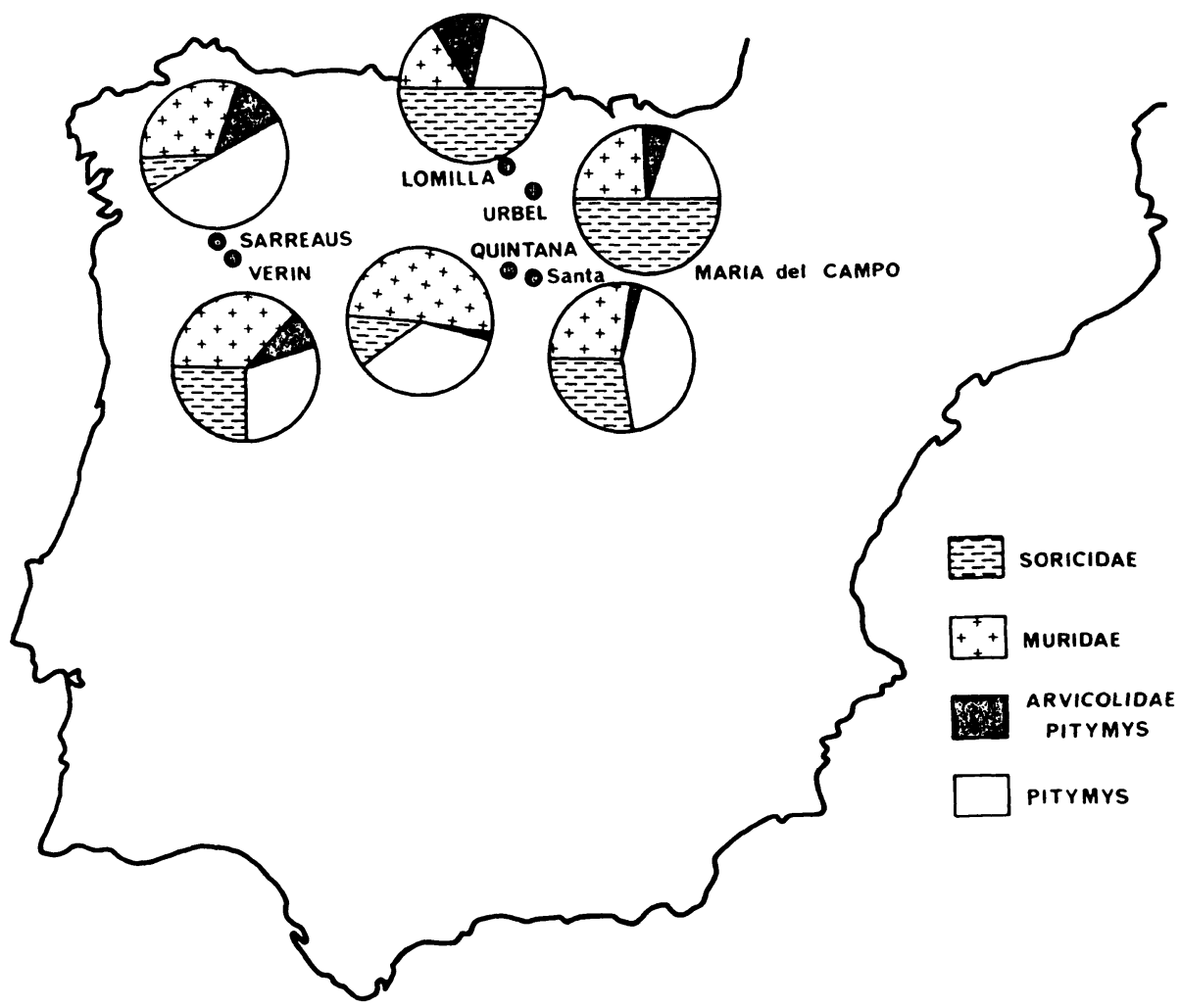

Fig. 1. - Répartition des stations étudiées et proportions des proies dans les pelotes de réjection.

TABLEAU 1. - Altitude et variables climatiques des stations.

Quintana $\begin{gathered}\text { Santa Maria } \\ \text { del Campo Urbel Lomilla }\end{gathered}$ Verin $\quad$ Sarreaus

$\begin{array}{lcccccc}\text { Altitude }(\mathrm{m}) & 750 & 850 & 980 & 900 & 612 & 660 \\ \begin{array}{l}\text { Température }\left({ }^{\circ} \mathrm{C}\right): \\ \text { moyenne de janvier }\end{array} & 2,3 & 2,8 & 2,3 & 2,3 & 4,7 & 4,7 \\ \begin{array}{l}\text { moyenne de juillet } \\ \text { 19,8 }\end{array} & 20,8 & 17,8 & 17,8 & 17,3 & 17,3 \\ \begin{array}{l}\text { Précipitation totale } \\ \text { annuelle (mm) }\end{array} & 481 & 431 & 628 & 628 & 893 & 893 \\ \begin{array}{l}\text { Type de climat : } \\ \text { méditerranéen }\end{array} & \text { tempéré } & \text { tempéré } & \begin{array}{c}\text { tempéré } \\ \text { frais }\end{array} & \begin{array}{c}\text { tempéré } \\ \text { frais }\end{array} & \begin{array}{c}\text { tempéré } \\ \text { frais }\end{array} & \begin{array}{c}\text { tempéré } \\ \text { frais }\end{array}\end{array}$


Les huit populations étudiées sont identifiées comme suit :

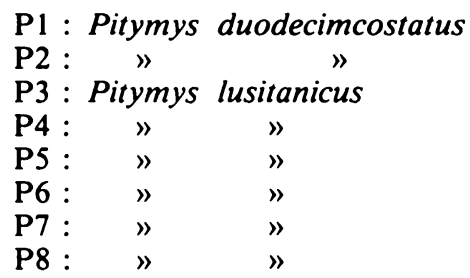

Quintana del Puente
Santa Maria del Campo
Quintana del Puente
Santa Maria del Campo
Urbel
Lomilla
Verin
Sarreaus

La détermination des $M_{1}$ associées à un crâne n'a pas posé de problèmes, mais pour pouvoir attribuer à l'une ou l'autre espèce les $M_{1}$ isolées de Quintana et Santa Maria del Campo, il a fallu mettre au point une fonction discriminante à partir des $M_{1}$ associées à un crâne de Quintana et Santa Maria del Campo.

\section{III - MORPHOMÉTRIE}

Pour standardiser les mesures on a défini un axe de symétrie qui passe par les bords externes de l'émail du premier et du quatrième angle rentrant. Vingt-trois mesures sont prises sur chacune des $M_{1}$, six sur l'ensemble de la dent, dix-sept sur le complexe antérieur (fig. 2 A-B). A partir de ces mesures, treize variables ont été calculées (fig. 2 C-D).

Les analyses statistiques portent sur ces variables, notées V1 à V13 :

V1 : Rapport entre les triangles internes et externes de la dent ( = dissymétrie des triangles dentaires).

V2 : Longueur maximale de la dent prise perpendiculairement à l'axe de symétrie.

V3 : Rapport entre le complexe antérieur et la longueur maximale de la dent.

V4 : Inclinaison du complexe antérieur par rapport à l'axe de symétrie.

V5 : Norme du vecteur W joignant les extrémités des triangles 4 et 5.

V6 : Rapport entre la norme du vecteur b joignant les angles rentrants 6 et 7 et la forme du vecteur W ( = fermeture du rhombe pitymyen).

V7 : Angle $\alpha 1$ fait par les vecteurs $b$ et $W$.

V8 : Norme du vecteur e joignant les extrémités des triangles 6 et 7 .

V9: Angle $\alpha 2$ fait par les vecteurs e et $W$.

V10 : Rapport entre la norme du vecteur d joignant les angles rentrants 8 et 9 et la norme du vecteur e ( $=$ ouverture du rhombe antérieur).

V11 : Angle $\alpha 3$ fait par les vecteurs d et $W$.

V12 : Rapport entre la norme du vecteur $f$ joignant les triangles 8 et 9 et la norme du vecteur e.

V13 : Angle $\alpha 4$ fait par les vecteurs $f$ et $W$.

\section{IV - VARIABILITÉ MORPHOLOGIQUE DES $\mathbf{M}_{1}$}

La figure 3 montre la variabilité morphologique des $\mathrm{M}_{1} \mathrm{D}$ de Pitymys duodecimcostatus et Pitymys lusitanicus des sites étudiés. Les $\mathrm{M}_{1} \mathrm{D}$ sont constituées d'une boucle postérieure, de trois triangles clos (T1, T2, T3), de deux triangles opposés et confluents (T4, T5) constituant le rhombe pitymyen plus ou moins ouvert dans un complexe antérieur, lui-même constitué de deux triangles opposés et confluents (T6, T7), plus ou moins ouverts dans une boucle antérieure ovoïde 


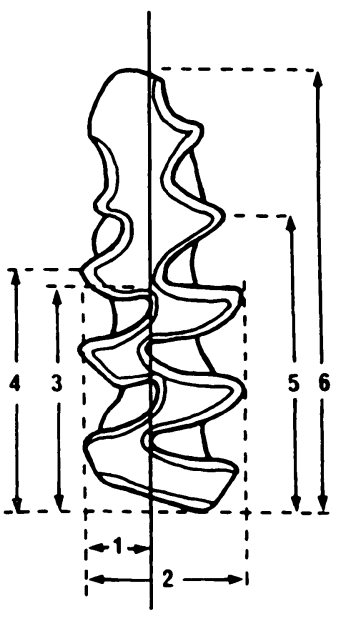

A

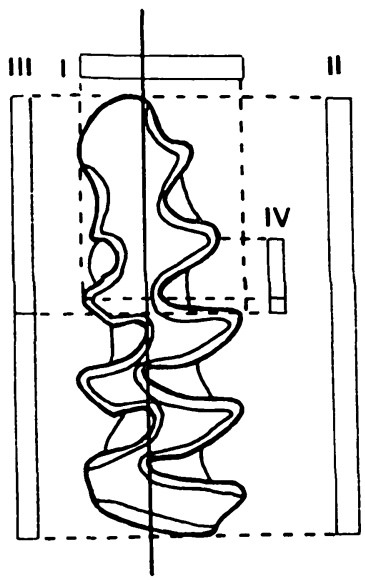

C
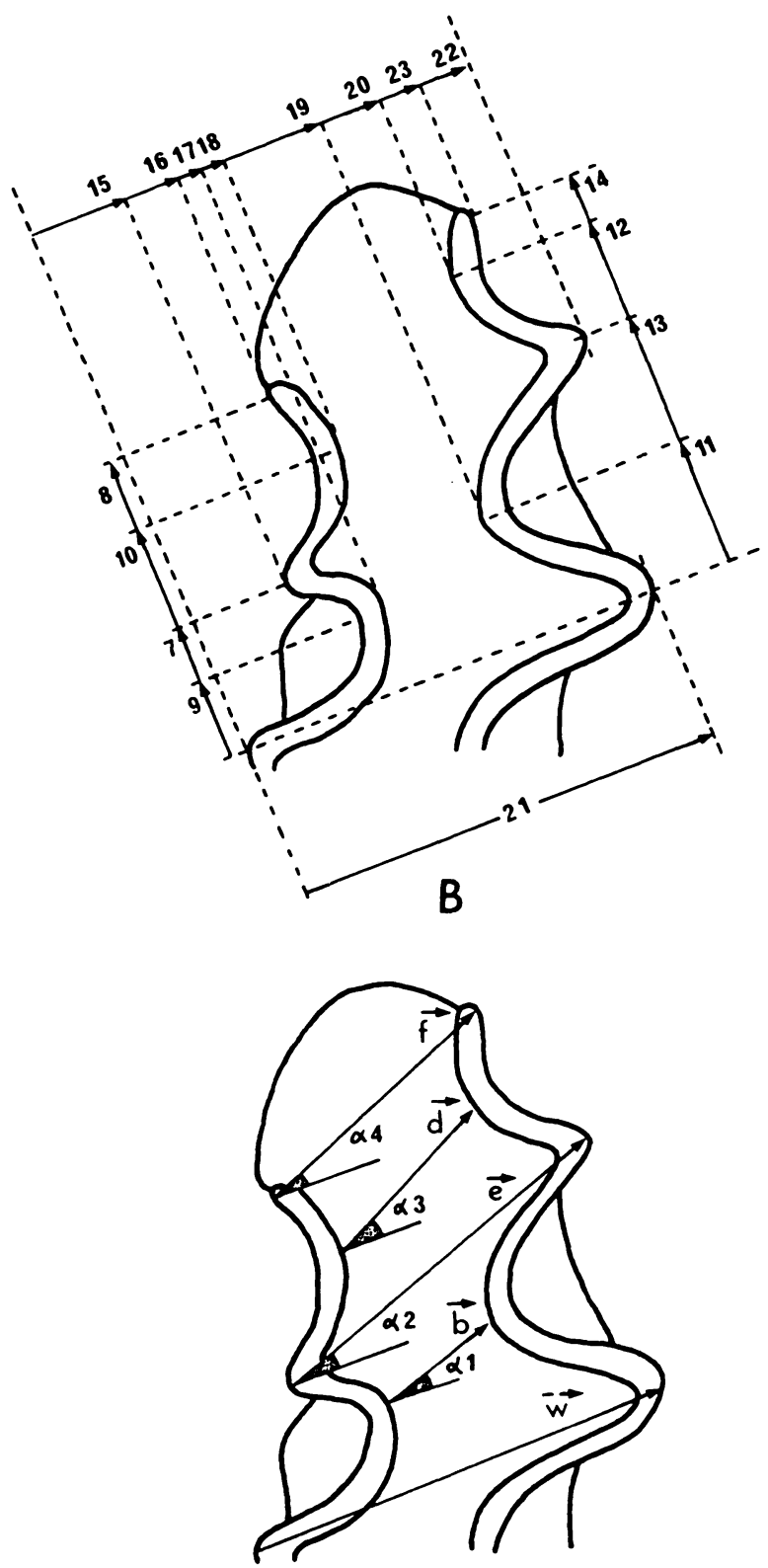

D

Frig. 2. - Morphométrie de la $\mathrm{M}_{1}$ des Pitymys. A : Axe de symétrie et mesures générales de la $M_{1}$. B : Mesures du complexe antérieur. C, D : Variables transformées (C : Visualisation des 4 premières variables; V1 $=\mathrm{I}, \mathrm{V} 2=\mathrm{II}, \mathrm{V} 3=\mathrm{III}, \mathrm{V} 4=\mathrm{IV}$ ). 

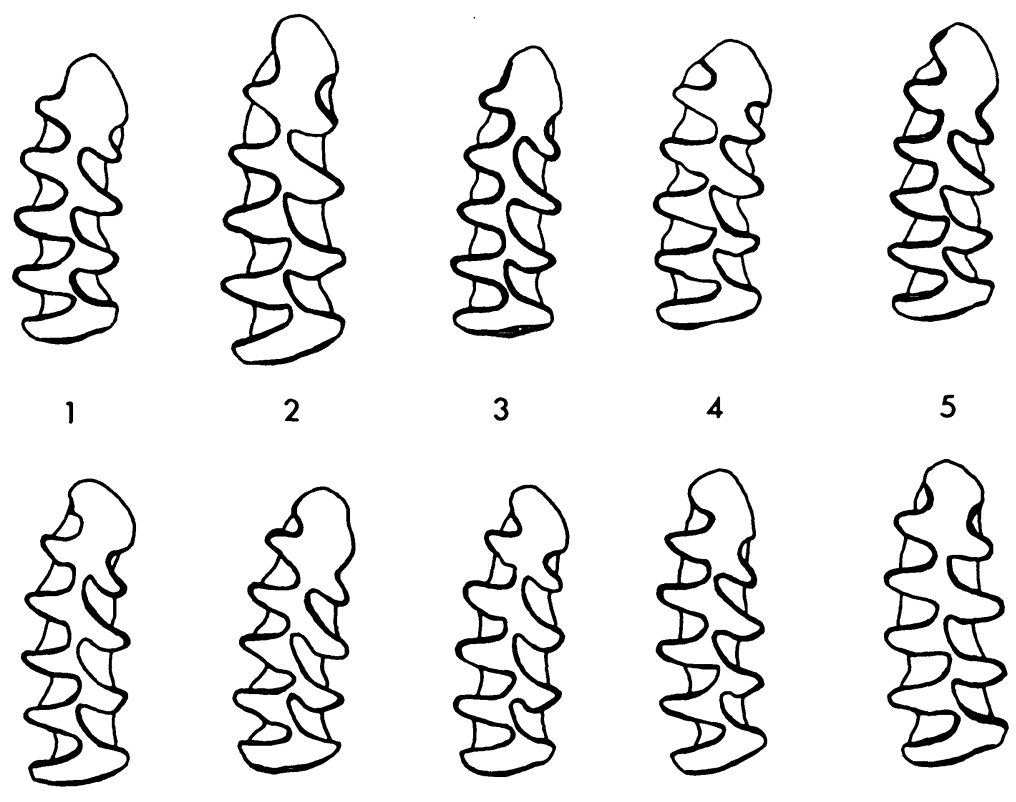

6

$$
7
$$

8

9

10
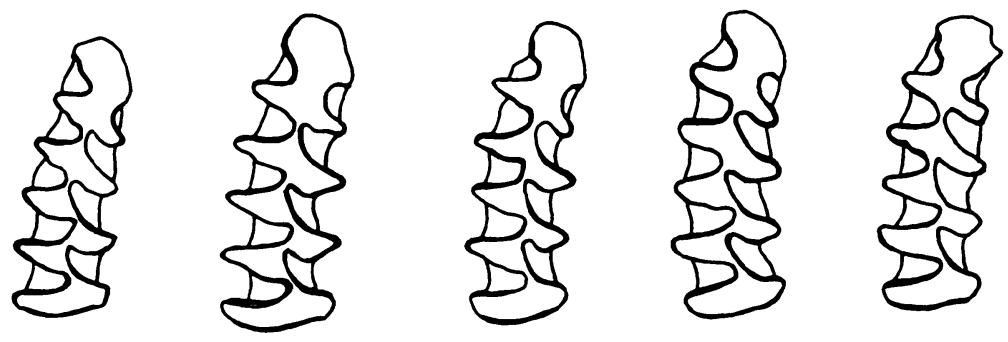

11

12

13

14

15
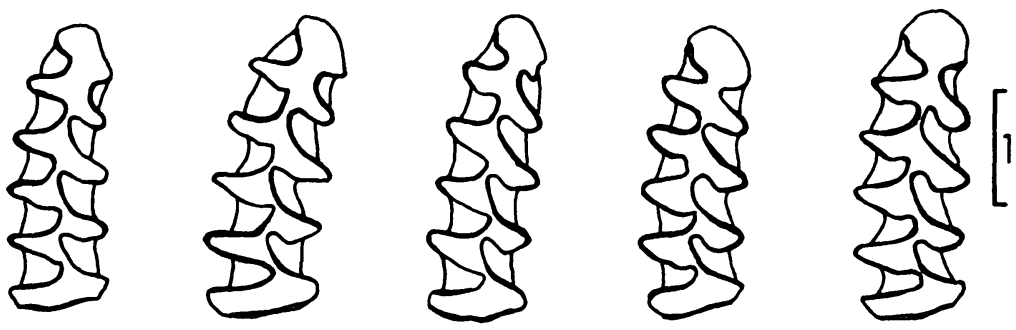

16

17

18

19

20

Fig. 3 
ou arrondie. L'isolement accentué des triangles T6-T7 de la boucle antérieure (fig. $3: 18,19,20$ ) crée un second rhombe pitymyen. La variabilité morphologique des Pitymys étudiés porte essentiellement sur ce complexe antérieur, sur l'isolement plus ou moins grand du second rhombe pitymyen avec la boucle antérieure. Chez Pitymys duodecimcostatus le second rhombe pitymyen est généralement peu développé et largement ouvert dans la boucle antérieure (fig. $3: 1,2,5$, 10) alors que chez $P$. lusitanicus il est mieux caractérisé, mieux isolé de la boucle antérieure (fig. $3: 11$ à 20). Lorsque $P$. lusitanicus n'est pas sympatrique avec $P$. duodecimcostatus, certaines $\mathbf{M}_{1}$ des deux espèces peuvent être confondues, car elles présentent des morphologies analogues.

\section{V - MÉthodes STATistiques}

\section{Statistiques univariées}

La moyenne et l'écart-type de chaque variable ont été calculés pour les huit populations.

\section{Analyses multivariées}

Les analyses multivariées sont maintenant fréquemment employées en biologie, aussi nous ne reviendrons pas sur l'aspect technique de ces méthodes (Lefèbvre 1980).

a) Analyse factorielle des correspondances. Cette analyse a notamment pour objet de voir graphiquement quelles sont les variables éventuelles qui caractérisent le mieux les populations étudiées.

b) Distances généralisées. Cette méthode a été utilisée pour tenter de répondre aux deux questions suivantes :

- pour une population donnée, la sous-population « dents droites » est-elle statistiquement différente de la sous-population "dents gauches "?

- pour une population donnée, quelles sont les populations qui lui sont les plus proches ou les plus éloignées, et ces populations sont-elles statistiquement différentes?

c) Fonction discriminante. L'objet du calcul d'une fonction discriminante entre deux populations est de trouver la combinaison linéaire des variables qui discriminent le mieux ces deux populations et de voir quelles sont les variables qui contribuent le mieux à cette discrimination. Le calcul de fonctions discriminantes a servi d'une part à attribuer les $M_{1}$ non associées à un crâne à l'une ou l'autre des deux espèces, et d'autre part à rechercher les variables qui contribuent le mieux à la discrimination de deux groupes donnés (exemple : $P$. duodecimcostatus et $P$. lusitanicus).

Fig. 3. - Variations morphologiques des $\mathrm{M}_{1} \mathrm{D}$ de Pitymys duodecimcostatus et lusitanicus (échelle $1=1 \mathrm{~mm}$ ).

1, P. duodecimcostatus : Santa Maria del Campo. 2, id. : Quintana del Puente. 3, $P$. lusitanicus: Santa Maria del Campo. 4, id. : Verin. 5, P. duodecimcostatus : Quintana del Puente. 6, id. : Santa Maria del Campo. 7, P. lusitanicus : Sarreaus. 8, id. : Verin. 9, id. : Sarreaus. 10, P. duodecimcostatus : Quintana del Puente. 11, P. lusitanicus: Quintana del Puente. 12, id. : Lomilla. 13-14, id. : Urbel del Castillo. 15, id. : Lomilla. 16-17, id. : Quintana del Puente. 18, id. : Lomilla. 19-20, id. : Verin. 


\section{VI - RÉSULTATS}

La fermeture du rhombe pitymyen (V6) et l'ouverture du complexe antéricur (V10) présentent une grande variabilité (tabl. 2). Alors que l'ouverture du complexe antérieur semble avoir une valeur discriminante entre les deux espèces, la fermeture du rhombe pitymyen ne semble pas avoir de valeur spécifique, mais présente une grande variabilité dans chacune des huit populations. Pour cette raison cette variable a été exclue des analyses multivariées.

\section{Position des $M_{1}$ non associées à un crâne}

La fonction discriminante entre les $M_{1}$ associées à un crâne de l'une ou l'autre espèce de Quintana et Santa Maria del Campo donne un pourcentage d'individus bien classés égal à cent. Cette fonction permet donc d'attribuer à $P$. lusitanicus ou $P$. duodecimcostatus avec un risque d'erreur théorique très faible (pratiquement nul) les $M_{1}$ isolées et ainsi de n'exclure pour l'étude aucune dent provenant de ces deux stations.

\section{Comparaison "dents droites-dents gauches"}

La comparaison «dents droites-dents gauches » à partir de l'analyse des distances généralisées ne montre dans chaque station aucune différence significative entre ces dents. En effet dans tous les cas on obtient un test $F$ de Fisher non significatif $(\mathrm{P}>0,05)$. Pour chacun des huit échantillons, il est donc possible de regrouper dans une même population les dents droites et gauches.

\section{Comparaison des deux espèces}

L'analyse factorielle des correspondances met en évidence une séparation partielle des deux espèces le long de l'axe 1 qui représente $40 \%$ de l'inertie (fig. 4 et 5). Les $P$. duodecimcostatus, tous situés sur la partie négative de l'axe, se caractérisent par une ouverture du complexe antérieur (V10) élevée et une inclinaison du complexe antérieur (V4) faible.

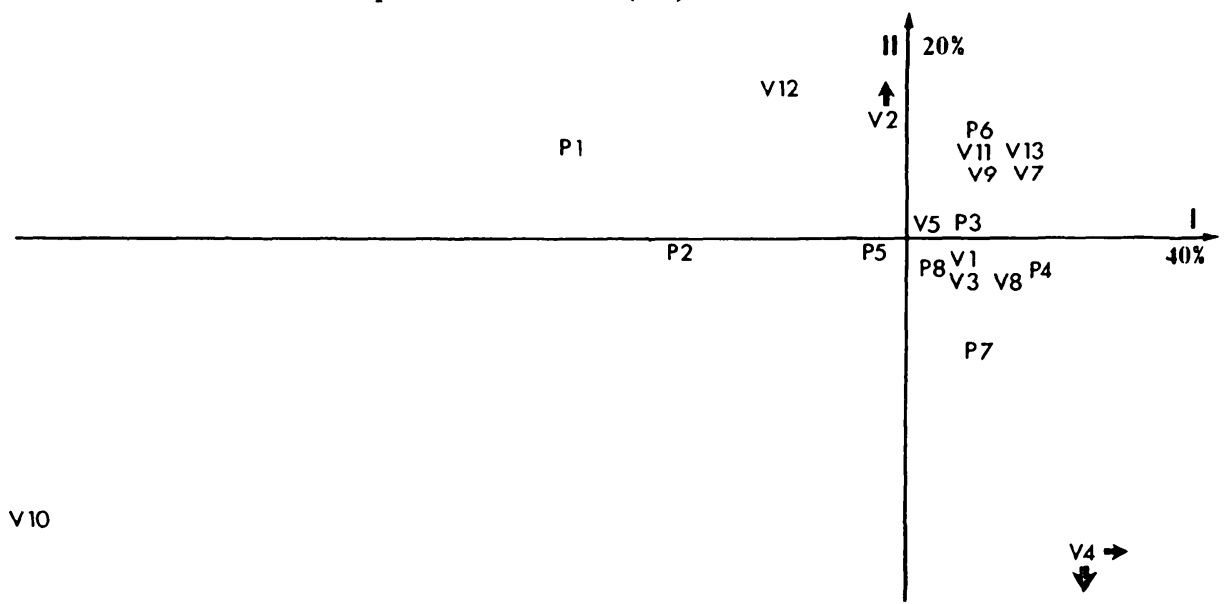

Fig. 4. - Analyse factorielle des correspondances des Pitymys duodecimcostatus et lusitanicus du N-W de l'Espagne. Projection des variables et des centres de gravité des populations dans le plan 1-2. 
A l'opposé, les $P$. lusitanicus de la zone de sympatrie, caractérisés par une ouverture du complexe antérieur faible et une inclinaison du complexe antérieur élevée, se situent tous sur la partie positive de l'axe. Certains $P$. lusitanicus, provenant de la zone d'allopatrie et qui présentent une morphologie dentaire

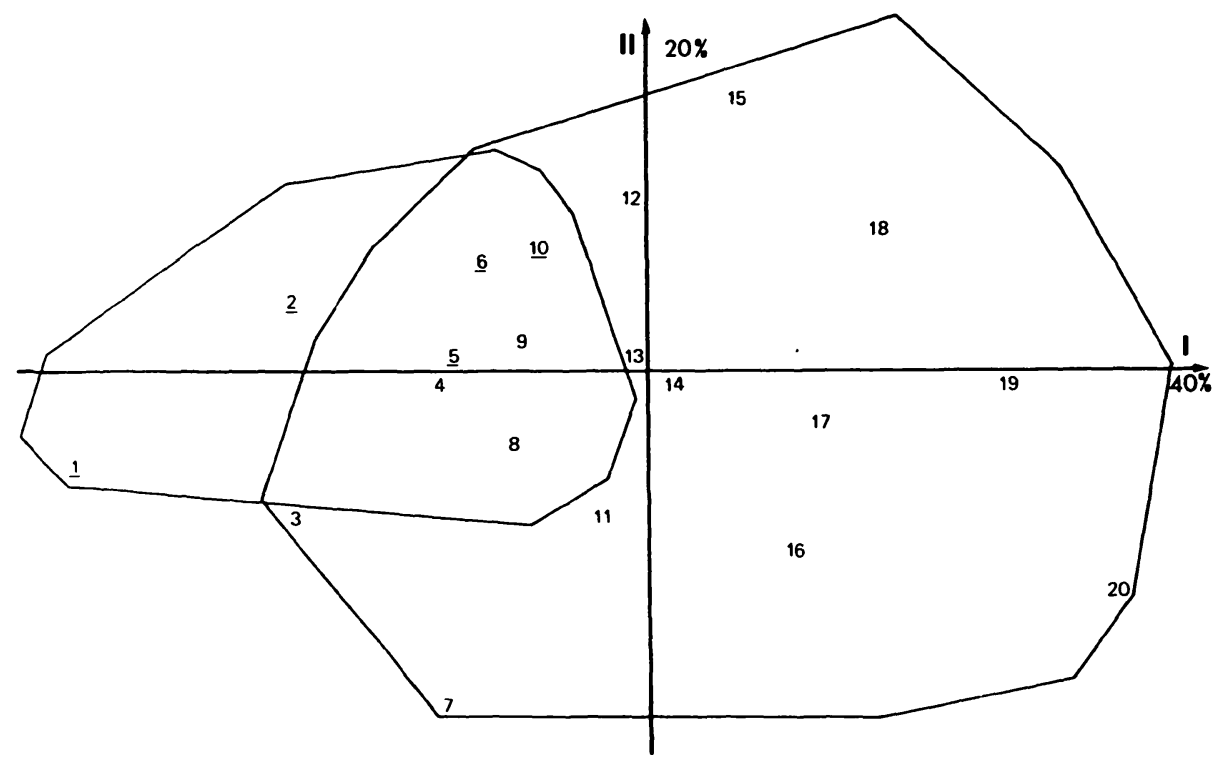

Fig. 5. - Analyse factorielle des correspondances : projection des nuages de 20 dents de $P$. lusitanicus et $P$. duodecimcostatus dans le plan 1-2. Pour le dessin des dents, se reporter à la figure 3 .

proche de celle de $P$. duodecimcostatus, se placent sur la partie négative de l'axe (fig. $3: 3,4,7,8,9,11,12,13)$. L'axe 2 (20\% de l'inertie) ne montre pas de discrimination des deux espèces.

L'analyse des distances généralisées et la fonction discriminante permettent de préciser ces observations. L'analyse des distances généralisées montre que chaque population de $P$. lusitanicus est statistiquement différente $(P<1 \%)$ de chaque population de $\boldsymbol{P}$. duodecimcostatus (fig. 6). Cette analyse montre aussi que les populations sympatriques de $\boldsymbol{P}$. lusitanicus ne sont pas plus proches des populations de $\boldsymbol{P}$. duodecimcostatus que les populations allopatriques de $P$. lusitanicus.

La fonction discriminante calculée pour l'ensemble des dents donne un pourcentage d'individus bien classés égal à 91 . Les deux variables les plus discriminantes sont respectivement l'ouverture du complexe antérieur (V10) et l'inclinaison du complexe antérieur (V4). Leur contribution respective est de $52 \%$ et $10 \%$.

\section{Comparaison des populations de P. lusitanicus entre elles}

La grande variation morphologique des dents de $P$. lusitanicus est illustrée par la position sur la figure 5 de 15 dents prises au hasard.

L'axe 1 de l'analyse factorielle des correspondances montre que seuls des individus de la zone d'allopatrie se situent dans le nuage de $P$. duodecimcostatus 


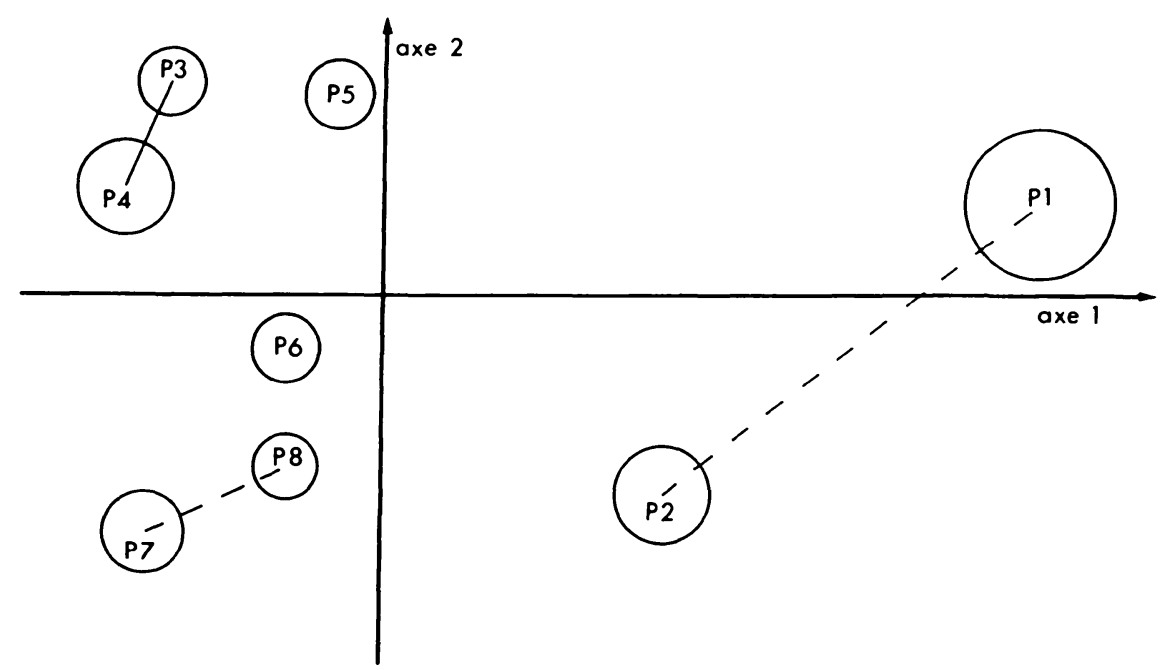

Fig. 6. - Analyse des distances généralisées : dispersion des centres de gravité (avec leur intervalle de confiance) dans les huit populations étudiées dans le plan 1-2. Droite continue : les deux populations ne sont pas statistiquement différentes à $5 \%$. Droite discontinue : les deux populations ne sont pas statistiquement différentes à $1 \%$. Pas de droite : les deux populations sont statistiquement différentes à $1 \%$.

(fig. 5). L'axe 2 de cette même analyse oppose la population de Lomilla (P6), caractérisée par une longueur de la dent (V2) élevée et une inclinaison du complexe antérieur (V4) faible, aux autres populations de $P$. lusitanicus (fig. 4).

L'analyse des distances généralisées montre que les six populations de $P$. lusitanicus peuvent se classer en quatre groupes :

- groupe 1: P3 et P4: populations de la zone de sympatrie. Elles ne sont pas statistiquement différentes à $5 \%(\mathrm{P}>5 \%)$;

- groupe 2: P7 et P8 : populations de Galice. Elles ne sont pas statistiquement différentes à $1 \%(5 \%>\mathrm{P}>1 \%)$;

- groupes 3 et $4:$ les populations de Lomilla et Urbel ne sont liées à aucune population $(P<1 \%)$.

Le calcul des fonctions discriminantes entre ces quatre groupes montre qu'il n'est pas possible de discriminer de façon satisfaisante l'un de ces groupes par rapport aux trois autres, le pourcentage d'individus bien classés étant au mieux de 73. Toutefois le calcul de ces fonctions discriminantes permet de voir quelles sont les variables qui caractérisent chaque groupe (tab. 3).

\section{Discussion}

L'ensemble des analyses faites montre que deux variables, l'ouverture du complexe antérieur (V10) et l'inclinaison du complexe antérieur (V4), ont un rôle essentiel dans la distinction des deux espèces. La longueur de la dent (V2), généralement plus grande chez $P$. duodecimcostatus, n'a pas dans cette étude une bonne valeur discriminante. En effet, les individus des deux espèces de Santa 


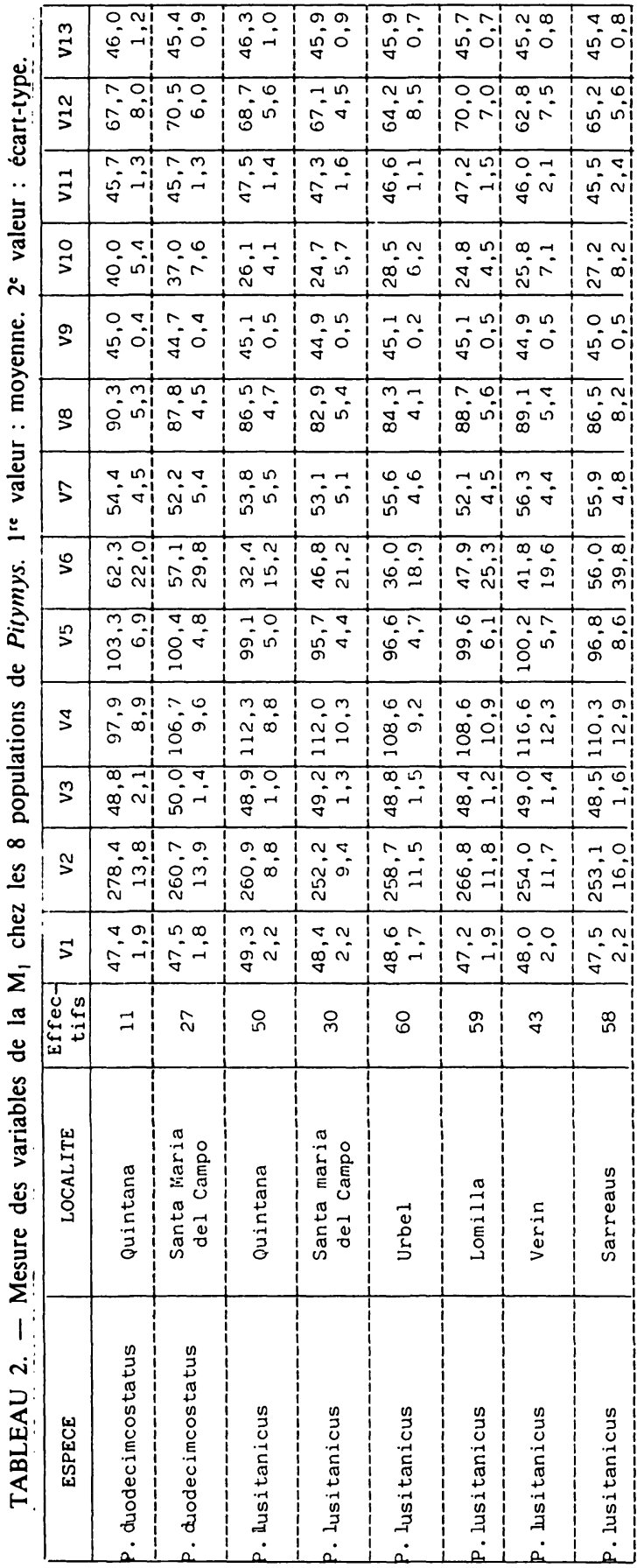


TABLEAU 3. - Comparaison des groupes de Pitymys lusitanicus. Variables caractérisant le mieux chacun des groupes d'après le calcul des fonctions discriminantes. Le qualificatif faible ou élevée indique si la variable est plus faible ou plus élevée dans le groupe considéré par rapport à l'ensemble des $P$. lusitanicus.

\begin{tabular}{|c|c|c|}
\hline Groupe & Variables & $\begin{array}{l}\text { Contribution de la variable dans la } \\
\text { fonction discriminante }\end{array}$ \\
\hline \multirow{3}{*}{$\begin{array}{l}\text { Quintana - } \\
\text { Santa Maria } \\
\text { del Campo }\end{array}$} & V8 faible & $24 \%$ \\
\hline & V13 élevée & $23 \%$ \\
\hline & V11 élevée & $17 \%$ \\
\hline \multirow[t]{3}{*}{ Verin-Sarreaus } & V2 faible & $33 \%$ \\
\hline & $V_{11}$ faible & $20 \%$ \\
\hline & V8 élevée & $15 \%$ \\
\hline \multirow{3}{*}{ Urbel } & V8 faible & $23 \%$ \\
\hline & $v_{12}$ faible & $21 \%$ \\
\hline & V10 élevée & $19 \%$ \\
\hline \multirow{3}{*}{ Lomilla } & élevée & 220 \\
\hline & V12 élevée & $17 \%$ \\
\hline & V8 élevée & $13 \%$ \\
\hline
\end{tabular}

Maria del Campo ont des dents de longueur réduite, notamment en comparaison de celles des individus de la station voisine de Quintana (tab. 2). Des conditions environnementales locales (climat ?) pourraient expliquer ce phénomène.

La proximité géographique des populations de $P$. lusitanicus ne suffit pas à expliquer à elle seule la variabilité observée. Les populations de Galice sont proches l'une de l'autre. De même la population de Quintana n'est pas statistiquement différente de celle de Santa Maria del Campo (analyse des distances généralisées : $\mathrm{P}<1 \%$ ). Le climat (tabl. 1) et les associations de faunes (fig. 1), semblables dans ces deux stations, n'expliquent pas une telle différence morphologique.

Par contre la plus grande variabilité morphologique observée chez les $P$. lusitanicus de Galice, pour l'ouverture du complexe antérieur (V10) (tabl. 2), par comparaison avec celle des $P$. lusitanicus de Lomilla, pourrait s'expliquer par une compétition interspécifique. Par exemple, Microtus arvalis, absent de Galice, pourrait être en compétition écologique avec $P$. lusitanicus à Lomilla.

Chez les Pitymys archaïques l'ouverture du complexe antérieur est élevée et l'inclinaison du complexe antérieur faible. $P$. lusitanicus présente une ouverture du complexe antérieur plus faible et une inclinaison du complexe antérieur plus élevée que $P$. duodecimcostatus. Ces observations semblent confirmer la descendance de $P$. lusitanicus à partir de $P$. duodecimcostatus (cladogenèse; Chaline 1966, 1972).

\section{VIII - CONCLUSION}

La présente étude de la morphométrie de la $\mathrm{M}_{1}$ chez les Pitymys du nordouest de l'Espagne semble confirmer que $P$. lusitanicus est issu de $P$. duodecimcostatus par cladogenèse. En effet, les deux variables : ouverture du rhombe T6 
et $\mathrm{T} 7$ et l'inclinaison du complexe antérieur, qui discriminent le mieux les deux espèces, peuvent être considérées comme un caractère apomorphe chez $P$. lusitanicus. Lorsque $\boldsymbol{P}$. lusitanicus n'est pas sympatrique avec $\boldsymbol{P}$. duodecimcostatus, ses populations présentent une plus grande variabilité des $\mathbf{M}_{1}$, certaines dents ayant alors une morphologie semblable à celle de $P$. duodecimcostatus. Ce déplacement de morphologie pourrait résulter du fait que $\boldsymbol{P}$. lusitanicus occupe alors une plus large niche écologique, ou de problèmes de compétition. La spéciation récente de $P$. lusitanicus et la bonne discrimination odontologique des deux espèces en sympatrie suggèrent une compétition écologique rigoureuse entre les deux espèces. Toutefois la seule compétition entre les deux espèces n'est peut-être pas le seul facteur permettant d'expliquer la variabilité des populations de $P$. lusitanicus, Microtus arvalis notamment pouvant entrer lui aussi en compétition écologique avec $P$. lusitanicus. Toutefois cette étude faite à partir de matériel provenant de pelotes de chouette effraie ne permet pas l'étude du rôle des facteurs écologiques (comme les années favorables ou défavorables pour la croissance des individus). Des études analogues devraient être entreprises sur des espèces de Pitymys appartenant aux groupes subterraneus et savii et sur des individus étudiés biochimiquement et caryologiquement, afin d'affiner la connaissance de l'évolution de ce groupe de mammifères.

\section{REMERCIEMENTS}

Nous tenons à remercier nos collègues B. Laurin et D.D. Rousseau (U.A. CNRS 157) pour l'utilisation des programmes d'analyses multivariées et Mme C. Fourcault qui a assuré la frappe, ainsi que, pour leur aide, le CSC et la CAICYT (programme 22107) d'Espagne et M. E. Collado.

\section{BIBLIOGRAPHIE}

AlmaçA, C., 1967. - Sur la position taxonomique de Pitymys lusitanicus (Gerbe) (Mamm. Rod.). Arg. Mus. Boc. (2a ser.), 1, $13: 249-256$.

AlmaÇA, C., 1973. - Sur la structure des populations des Pitymys ibériques. Rev. Fac. Ciencias Lisboa (2a ser.), C, XVII, $2^{\mathrm{e}}: 383-426$.

Brochet, G., en prép. - Evolution du groupe des Microtus en Eurasie. Thèse $3^{\mathrm{e}}$ cycle, Univ. Dijon (France).

Chaline, J., 1966. - Un exemple d'évolution chez les Arvicolidés (Rodentia) : les lignées Allophaiomys, Pitymys et Microtus. C.R. Ac. Sc. Paris, D, 263 : 1022-1024.

Chaline, J., 1972. - Les rongeurs du Pléistocène moyen et supérieur de France. Cahiers de Paléontologie, Ed. CNRS, Paris : 1-410.

Elias Castillo, F., et L. Ruiz-Beltran, 1977. - Agroclimatologia de España. Cuaderno I.N.I.A., Ministerio de Agricultura, Madrid, $\mathrm{n}^{\circ} 7$.

Ellerman, J.R., et T.C.S. MORRISON-SCOTT, 1951. - Checklist of Palaeartic and Indian Mammals, 1758 to 1946. British Mus. Nat. Hist., London. 
Heim de Balsac, H., et F. DE Beaufort, 1967. - La spéciation des Pitymys galloibériques. Une nouvelle espèce pour la faune de France. Mammalia, 31 : 367-380.

LEFEBVRE, J., 1980. - Introduction aux analyses statistiques multidimensionnelles. Masson, Paris, $259 \mathrm{p}$.

Meylan, A., 1970. - Caryotypes et distribution de quelques Pitymys européens (Mamm. Rod.). Rev. Suisse Zool., 77 : 562-575.

Niethammer, J., et F. KRAPP, 1982. - Handbuch der Säugetiere Europas. Bd 2/1, Rodentia II. Akad. Verl. Ges. Wiesbaden.

SPITZ, F., 1978. - Etude craniométrique du genre Pitymys. Mammalia, 42 : 267-304.

Winking, H., et J. Niethammer, 1970. - Der Karyotyp der beiden Bleinen, iberischen Pitymys Arten (Mamm., Rod.). Bonn. Zool. Beitr., 21 : 284-289. 